\title{
Evidência de Validade de Construto para o Teste de Atenção Sustentada
}

\author{
Evidence of construct validity for \\ the teste de atenção sustentada \\ Evidencia de validez de constructo para \\ la prueba de atención sostenida
}

Fabián Javier Marin Rueda, Ana Paula Porto Noronha, Fermino Fernandes Sisto \& Daniel Bartholomeu

Universidade São Francisco
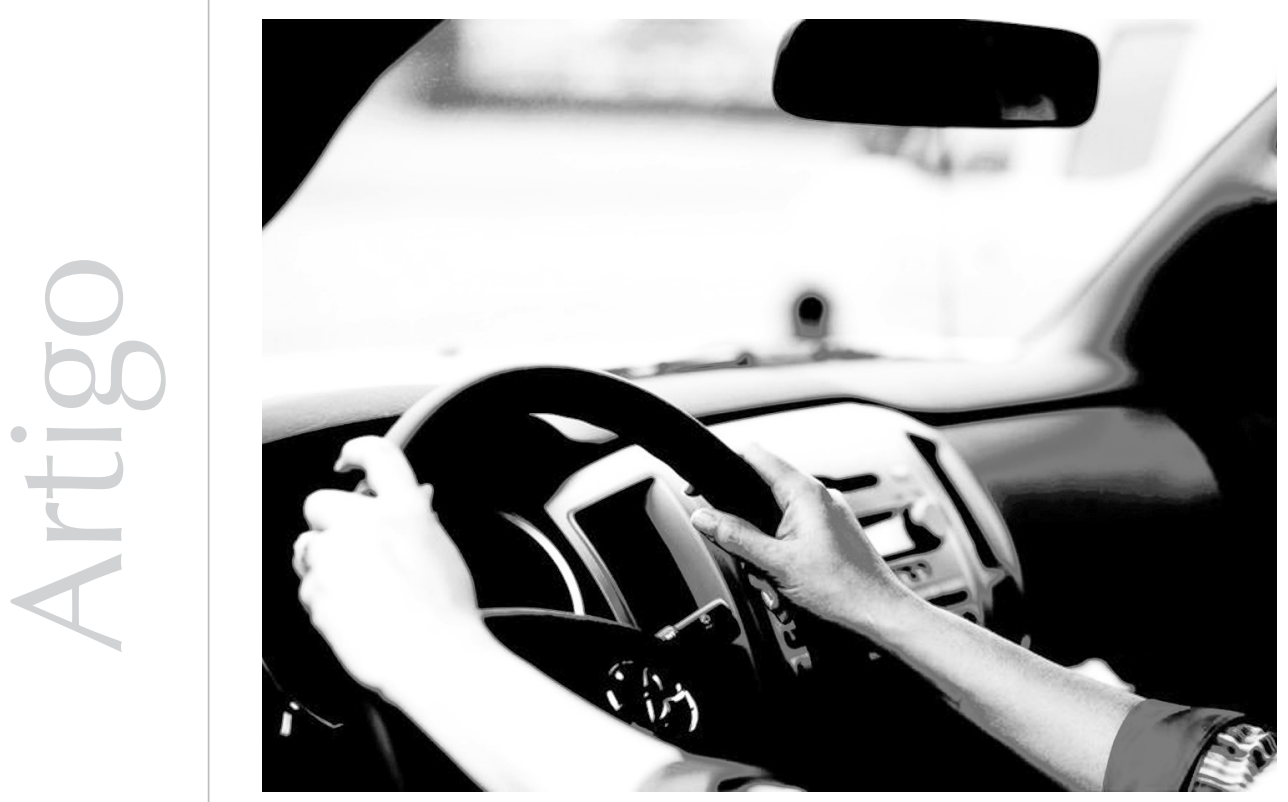
Resumo: O objetivo do estudo foi verificar evidência de validade de construto para o Teste de Atenção Sustentada. Participaram da pesquisa 127 indivíduos, que passaram pelo processo de avaliação psicológica para a obtenção da Carteira Nacional de Habilitação em clínicas credenciadas pelo DETRAN. Os resultados mostraram correlações negativas e significativas entre as medidas de concentração e de velocidade com qualidade do Teste de Atenção Sustentada e a idade. Nas análises de variância, o agrupamento das diferentes idades apresentou diferenças estatisticamente significativas tanto em relação à concentração como à velocidade com qualidade. Com base nesses resultados, concluiu-se que houve evidência de validade de construto, a partir da análise das idades, para essas medidas.

Palavras-chave: Atenção. Teste de Atenção Sustentada. Validade. Avaliação psicológica.

Abstract: This study aimed to verify evidence of construct validity for the Teste de Atenção Sustentada. 127 individuals, officially approved by the psychological assessment for attainment of their licenses to drive, participated in the research. The results showed negative and significant correlations among the measures of the Teste de Atenção Sustentada, which is Concentration and Speed with Quality of the response, with the ages. The variance analysis showed that the differences of the means in relation to the ranges of ages were statistically significant as well as between the measures of Concentration and Speed with Quality. On the basis of these results, the evidence of construct validity, by age analysis for these measures was accepted.

Keywords: Attention. Teste de Atenção Sustentada. Validity. Psychological assessment.

Resumen: El objetivo del estudio fue verificar evidencia de validez de constructo para la Prueba de Atención Sostenida. Participaron en la pesquisa 127 individuos, que pasaron por el proceso de evaluación psicológica para la obtención del Permiso Nacional de Conducir en clínicas acreditadas por el DETRAN. Los resultados mostraron correlaciones negativas y significativas entre las medidas de concentración y de velocidad con calidad de la Prueba de Atención Sostenida y la edad. En los análisis de varianza, la agrupación de las diferentes edades presentó diferencias estadísticamente significativas tanto con relación a la concentración como a la velocidad con calidad. Con base en esos resultados, se concluyó que hubo evidencia de validez de constructo, del análisis de las edades, para ésas medidas.

Palabras-clave: Atención. Prueba de Atención Sostenida. Validez. Evaluación psicológica.

O construto atenção apresenta vários conceitos e definições, podendo ser destacada a definição de Sternberg (2000), que a define como o "fenômeno pelo qual o ser humano processa ativamente uma quantidade limitada de informações, do enorme montante de informações disponíveis através dos órgãos dos sentidos, de memórias armazenadas e de outros processos cognitivos" (p. 78). Para o autor, a atenção refere-se à capacidade de, e ao esforço exercido para focalizar e selecionar um estímulo para ser processado, que leva o indivíduo a responder a determinados aspectos do ambiente em lugar de fazê-lo em relação a outros, e permite ao ser humano utilizar seus recursos cognitivos para emitir respostas rápidas e adequadas mediante estímulos que julgue importantes.

Por sua vez, Silva (1999) afirma que toda atividade mental precisa de direcionamento e seletividade. Assim, para o autor, a capacidade atencional seria a função cerebral responsável pela escolha dos estímulos que representam o foco de maior interesse em um dado momento e que possuiria grande importância para a realização de determinadas tarefas. Luria (1979) entende que a seleção dos estímulos e o direcionamento da atividade mental seriam mediados também pela importância e pelo 
Zillmer e Spiers

(1998) ressaltaram

que muitas vezes

a atenção seletiva

é utilizada como sinônimo da atenção concentrada, podendo ser

definida como a

capacidade de centrar-se em um estímulo importante, enquanto se suprimem

deliberadamente interesse por uma determinada tarefa em detrimento de outras.

Destaca-se que, embora as teorias e definições sobre atenção sejam amplas, não há consenso entre os pesquisadores em relação à melhor definição para o construto. Assim, muitas vezes, o que se aplica são definições operacionais que poderiam negligenciar alguns aspectos relevantes ao estudo do construto em questão. Nesse sentido, segundo Brickenkamp (2004), enquanto na Alemanha o termo mais utilizado é atenção seletiva, nos Estados Unidos, é mais comum "atenção seletiva e sustentada". Lezak (1995) afirma, ainda, que o teste $\mathrm{d} 2$, a título de exemplo, é conhecido como Teste de Concentração ou de Atenção Seletiva, enquanto, nos Estados Unidos, recebe o nome de Teste de Amplitude Atencional, de Atenção Seletiva ou de Atenção Sustentada. Por sua vez, Zillmer e Spiers (1998) ressaltaram que muitas vezes a atenção seletiva é utilizada como sinônimo da atenção concentrada, podendo ser definida como a capacidade de centrar-se em um estímulo importante, enquanto se suprimem deliberadamente outros estímulos distratores.

Dessa forma, com base nas distintas funções e características, diferentes classificações foram propostas ao longo dos anos sobre os diversos tipos de atenção, sendo também verificadas divergências em tais classificações. Em relação à atenção sustentada, ela é definida por Sisto, Noronha, Lamounier, Rueda e Bartholomeu (2006) como a capacidade de manter a vigilância e a observação por um longo período de tempo ou durante tarefas que "produzem tédio". Dessa forma, tal como defendido por Gaddes e Edgell (1994), quando uma pessoa é incapaz de manter a atenção focada em um determinado trabalho até o final, podese supor que ela apresente algum problema de atenção sustentada. No exercício de dirigir um veículo automotor, a avaliação do construto da atenção sustentada é de grande valia, pois informa sobre a possibilidade de distração do condutor.

Ao lado disso, a idade também tem sido uma variável bastante pesquisada no ambiente do trânsito, uma vez que ela se apresenta, muitas vezes, como determinante para o envolvimento ou não em acidentes de trânsito. Em âmbito estrangeiro, as pesquisas com motoristas encontram-se em estágios mais avançados de evolução teórica e metodológica, sendo que, dentre elas, a avaliação do impacto de treinamento para motoristas tem se destacado, uma vez que é necessária a implementação de diferentes medidas para a diminuição do número de acidentes de trânsito. Assim, é pertinente o estudo de Potvin, Champagne e LabergeNadeau (1988), uma vez que investigou os indicadores de segurança em estrada, o risco de acidente (com e sem danos) durante o primeiro ano de experiência na direção, a taxa de mortalidade/morbidez por acidente que envolvia pelo menos um motorista em seu primeiro ano de experiência na direção, o número de novos motoristas habilitados e a idade média de habilitação. Foram obtidos registros mensais sobre o número de acidentes e os danos que envolviam motoristas agrupados por sexo, idade, experiência e tipo de veículo (mantidos para análise apenas acidentes que envolviam pelo menos um motorista novo licenciado que dirigia um carro particular). Em acréscimo, foram coletados registros mensais sobre o número de novos motoristas habilitados e a idade média.

Os resultados da pesquisa de Potvin et. al. (1988) indicaram que há mais risco de 
acidentes em homens entre 16-17 anos do que entre 18-25 anos. Mas, possivelmente, a revelação mais oportuna desse trabalho tenha sido a de confirmar outros trabalhos realizados para avaliar programas de treinamento de motorista. Os autores chamaram a atenção para o fato de que, entre todas as medidas possíveis para melhorar a segurança em estradas, os programas de treinamento de motorista estavam entre os menos eficientes em termos de redução de acidentes, embora eles ainda fossem muito populares no mundo inteiro.

Preusser, Williams e Lund (1985) afirmaram que quase metade do total de acidentes com vítimas fatais ocorreu com motoristas que tem idade entre 16-19 anos, o que vai de encontro aos resultados do estudo anteriormente citado. Os autores enfatizaram, ainda, que os adolescentes, além de estarem incluídos em taxas elevadas de acidentes fatais, também apresentavam maior índice de responsabilidade nos acidentes nos quais se envolveram.

Ainda no que se refere à idade dos motoristas, de acordo com índices americanos, estaria havendo um aumento no número de pessoas idosas na direção de veículos (Kington, Reuben, Rogowski, \& Lillard, 1994), o que justifica a relevância de estudos com as faixas etárias com mais idade. Nesse sentido, a diferença entre motoristas novos e experientes no que se refere à distribuição de atenção visual sob diferentes níveis de estratégias cognitivas impostas por tipos de pista foi investigada por Crundall e Underwood (1998). A tarefa exigia que os motoristas dirigissem por 20 minutos em pistas com características diferentes, ao mesmo tempo em que os movimentos de seus olhos eram gravados. Foram encontradas diferenças entre os motoristas novos e experientes de acordo com o tipo de pista na qual dirigiram. Os resultados sugeriam que os experientes selecionaram as estratégias visuais de acordo com a complexidade da rodovia, enquanto os novos foram mais inflexíveis diante das necessidades de mudança. Dessa forma, os autores concluíram que o foco de atenção dos motoristas experientes e novos seria diferente.

Ainda no que se refere ao tema, a fim de determinar se havia diferença de idade especificamente associada com a habilidade de executar simultaneamente duas tarefas e de avaliar se as perdas cognitivas se correlacionavam com as diferentes situações propostas, Ribaupierre e Ludwig (2003) estudaram 81 jovens e 96 idosos, que foram submetidos a nove tarefas que se propunham a avaliar a capacidade atencional, administradas em condições simples e duais. Os resultados indicaram diferenças de desempenho em todas as tarefas e condições nas várias faixas etárias. Entretanto, a grande perda cognitiva em idosos, avaliada pela interação idade versus condição, foi observada somente em quatro das nove tarefas. Além disso, as correlações entre as tarefas duais e entre os escores das perdas cognitivas foram muito baixas quando a idade foi parcialmente controlada, e todas as tarefas duais produziram uma perda adicional em relação às simples, conforme o esperado.

Em acordo com os dados anteriores, o estudo de Anderson, Lidaka, Cabeza e Craik (2000) avaliou a relação entre idade e a atenção. Os sujeitos foram 17 indivíduos de 21 a 31 
anos e 12 de 63 a 76 anos. A tarefa consistiu de uma lista de 20 pares de palavras moderadamente relacionadas, como, por exemplo, dentista-luva. Os estímulos eram apresentados por computador e o sujeito era solicitado a ler a segunda palavra do par e a fazer uma imagem visual dessa palavra para facilitar a memorização. Os resultados revelaram que a performance na atenção estaria relacionada à idade, sendo que adultos jovens apresentaram melhor desempenho que adultos mais velhos. Assim, os autores destacaram que o curso da idade normal estaria associado a uma diminuição da capacidade de atenção, seja ela dividida, seja sustentada.

A performance em atenção sustentada em relação a diferentes idades foi também investigada por Berardi, Parasuraman e Haxby (2001) em 62 sujeitos, sendo 21 jovens, 21 de meia idade e 20 idosos saudáveis, tendo sido verificado não haver diferenças entre as idades na capacidade de atenção sustentada. Os autores ressaltaram, em que pesem as pontuações em atenção sustentada terem sido semelhantes ao longo dos grupos de idade, que foi observada uma diminuição dessa pontuação nos sujeitos mais velhos.

Também com o intuito de investigar o efeito da idade, do sexo e da educação sobre a atenção sustentada, Chan (2001) realizou uma pesquisa utilizando o The Sustained Attention to Response Task (SART) em um grupo contraste e outro, de pacientes com lesões cerebrais. Dois estudos foram realizados, sendo que, no primeiro, recrutou-se um grupo contraste para avaliar a potência do impacto da idade, educação e sexo no SART. O segundo estudo objetivou estabelecer validade discriminativa para o SART, sob a performance da atenção, em uma amostra de pacientes e não pacientes com lesão cerebral. A potência do impacto de idade, sexo e educação encontrada nas tarefas do SART foi mínima. Em contrapartida, o SART mostrou-se capaz de discriminar os pacientes com lesão cerebral daqueles que não a apresentavam.

Quanto às pesquisas de atenção sustentada em motoristas, Campagne, Pebayle e Muzet (2004) ressaltaram que, ao dirigir por muito tempo, monotonamente e à noite, a maioria dos motoristas mostra progressivamente sinais de fadiga visual e de perda da atenção sustentada. Sua capacidade de manter um desempenho adequado ao dirigir é comumente afetada e varia em razão da idade do motorista. Partindo desses pressupostos, os autores propuseram um estudo para verificar os efeitos da fadiga e da idade dos motoristas sobre a sua performance quando estão dirigindo. Foram investigados 46 motoristas, divididos em três categorias de idade, quais sejam, 20-30, 40-50 e 6070 anos. Os participantes percorreram uma via de 350 km à noite, em um simulador de direção. Os erros foram medidos em termos do número de incidentes e de desvios de velocidade alta. A evolução do nível de vigilância fisiológica foi avaliada por meio do eletroencefalograma, e, nos motoristas mais velhos, a diminuição da performance de dirigir foi correlacionada com a evolução do baixo nível de vigilância. Contrariamente, nos motoristas jovens e de meia idade, a deterioração do nível de vigilância atestado pela eletroencefalografia correlacionou-se com o aumento na gravidade dos erros.

Lucidi, Devoto, Bertini, Braibanti e Violani (2002) compararam o nível de atenção 
sustentada de um grupo de 59 motoristas jovens em dois momentos, das $9 \mathrm{~h}$ às $11 \mathrm{~h}$ da manhã e das $2 \mathrm{~h} 30 \mathrm{~m}$ às $5 \mathrm{~h}$, em um domingo. Os resultados indicaram uma queda da vigilância durante a madrugada, maior em sujeitos que tinham consumido mais álcool. Os estudantes que passaram o sábado à noite em discotecas mostraram maior decréscimo na vigilância até mesmo quando o efeito do consumo de álcool foi controlado por análise de covariância. O efeito das atividades sociais do sábado sobre os níveis de vigilância também se associou a diferentes períodos de tempo gasto quando acordados bem como a diferente tempo em que os sujeitos desempenharam a tarefa.

Se, por um lado, pode ser percebido que a avaliação da atenção sustentada em motoristas é freqüentemente encontrada em pesquisas realizadas no exterior, por outro, esse fato não vale para o Brasil. Ao lado disso, instrumentos que se proponham a mensurar tal construto nessa população parecem ser escassos, e até inexistentes no País. Dentro desse contexto, o presente estudo objetivou buscar evidência de validade de construto por meio da verificação da performance ao longo de diferentes idades para as medidas de concentração e de velocidade com qualidade do Teste de Atenção Sustentada (Sisto et al., 2006), desenvolvido para o contexto do trânsito.

\section{Método}

\section{Participantes}

Participaram da pesquisa 127 candidatos à obtenção da Carteira Nacional de Habilitação $(\mathrm{CNH})$, com idades que variavam de 20 a 47 anos $(M=28,13, D P=7,17)$, de diferentes cidades do Estado de Minas Gerais. Em relação ao sexo, 71 (55,9\%) eram homens e $56(44,1 \%)$ mulheres. Quanto à escolaridade, ela variou desde o ensino fundamental incompleto até a pós-graduação. As profissões dos participantes eram diversificadas.

Instrumento

Teste de Atenção Sustentada - AS (Sisto et al., 2006).

O teste é composto por 25 fileiras com 25 estímulos cada uma. O sujeito deve assinalar apenas um tipo de estímulo dentre as possibilidades. Para isso, o participante tem 15 segundos para completar cada fileira, sendo que, ao término do tempo estabelecido, o aplicador diz "próxima", e, dessa forma, o participante passa imediatamente para a linha seguinte e recomeça. Em média, o tempo de aplicação é de 10 minutos.

Nesse instrumento são extraídas três medidas, a saber, concentração, velocidade com qualidade e sustentação. A concentração refere-se à soma de itens que deveriam ser assinalados (tarefa solicitada), subtraídos os erros e as omissões. A velocidade com qualidade diz respeito à quantidade de itens que o indivíduo preencheu (tarefa solicitada acrescida de itens que não deveriam ser assinalados e não o foram), menos os erros e as omissões. Para classificar o nível de sustentação da pessoa, somam-se, das três primeiras linhas, os itens que deveriam ser marcados e o foram com os itens que não deveriam ser marcados e não o foram. Desse total, a soma de erros com omissões é subtraída. O mesmo procedimento é repetido com as três últimas linhas. Obtidos esses dois índices, subtrai-se o segundo do primeiro e o resultado é interpretado conforme as tabelas de normatização do manual, sendo verificado posteriormente 
se a pessoa mantém, perde ou aumenta a sustentação.

Quanto às propriedades psicométricas do teste, a evidência de validade concorrente foi comparada com o Teste Conciso de Raciocínio (Sisto, 2006) e com o Teste de Atenção Concentrada - AC (Cambraia, 2004). Também foi verificada evidência de validade discriminante quando comparada com o psicodiagnóstico miocinético - PMK (Mira, 2004).

No que tange à precisão, ela foi estudada pelo alfa de Cronbach, o coeficiente de consistência interna e o método das metades, sendo que, por este último, foram fornecidos os resultados pelos procedimentos de Guttman, SpearmanBrown e alfa das duas metades, para cada faixa etária e no geral. De uma forma geral, os coeficientes de precisão variaram de 0,73 até 0,97. Também foram consideradas as linhas ímpares como um subteste e os pares como outro, e os coeficientes variaram de 0,90 até 0,98. Por esses dados, os autores concluíram que o teste apresenta bons índices de precisão.

\section{Procedimento}

Após assinatura do Termo de Consentimento Livre e Esclarecido, deu-se início à coleta de dados. Vale ressaltar que todas as aplicações foram feitas por psicólogos que possuíam curso de perito examinador credenciado pelo DETRAN, e foram realizadas em salas isoladas e com espaço físico adequado.

\section{Resultados}

Num primeiro momento, foram realizadas estatísticas descritivas com as medidas trabalhadas neste estudo, quais sejam, a concentração e a velocidade com qualidade do AS e, em seguida, foi realizada uma correlação entre o AS e a idade. Foram verificadas, ainda, diferenças entre as faixas etárias por meio da análise de variância (ANOVA).

No caso da concentração, as pontuações variaram de -45 até $72(M=22,29$, $\mathrm{DP}=26,48)$, sendo a moda 50 pontos, e a mediana, 25. Verificou-se que houve uma concentração entre -5 e 45 pontos (68,5\%). Os participantes que apresentaram entre -45 e -4 pontos foram 18,9\%, e, entre 46 e $72,12,6 \%$. No caso da velocidade com qualidade, as pontuações variaram de 20 até 150, sendo a pontuação média 98,54 $(D P=26,83)$. A moda foi 124 , e a mediana, 102. A concentração de pontos ficou entre 100 e 130 (47,3\% da amostra).

Com o objetivo de verificar a relação entre as medidas de concentração e velocidade com a qualidade e a idade, foi realizada uma correlação entre essas variáveis. Os resultados evidenciaram uma correlação negativa e significativa $(r=-0,38$ e $r-=0,37)$ para as medidas de concentração e velocidade com qualidade, respectivamente. Esses dados indicaram que, conforme aumentou a idade, diminuiu a capacidade de concentração e de velocidade com qualidade, o que evidenciou uma relação entre a idade e essas medidas. Verificou-se também que essa relação foi muito semelhante para ambas as medidas do AS. Na Figura 1, é possível observar a distribuição dos participantes por idade na medida de concentração. 


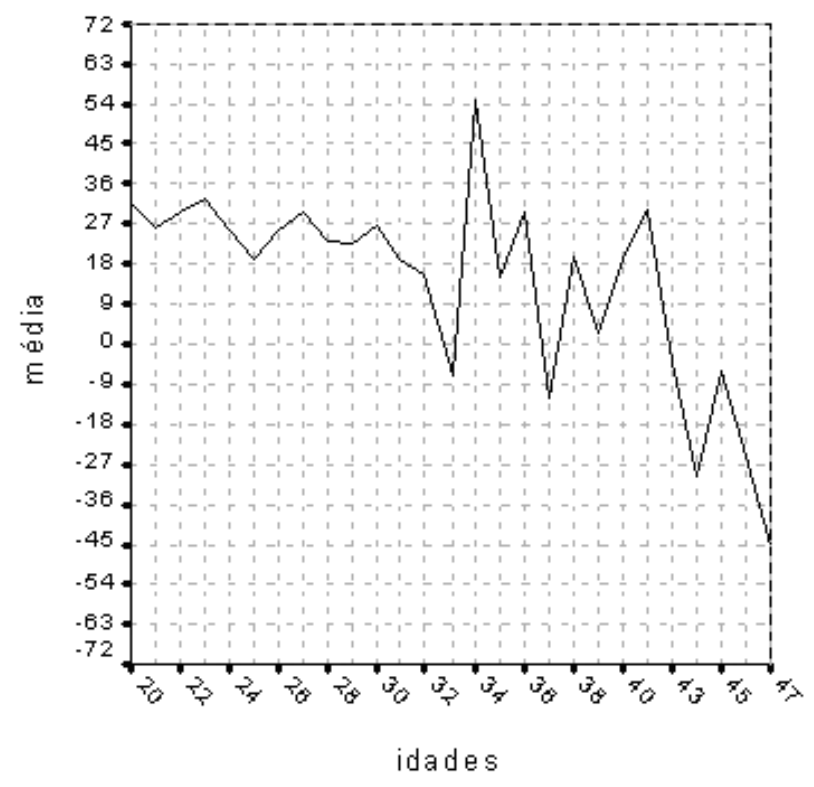

Figura 1. Média das pontuações na medida concentração no AS por idade dos participantes.

No manual do Teste de Atenção Sustentada, os autores apresentam seis faixas etárias que foram diferenciadas pela análise de variância, quais sejam, indivíduos de 18 e 19 anos, de 20 até 27 anos, de 28 até 36, de 37 até 41, de 42 até 55 anos e, por fim, pessoas com mais de 56 anos. Foi com base nesses dados que, neste estudo, procurou-se agrupar as idades; assim, foram formadas três faixas etárias, a saber, pessoas com até 27 anos (55,9\%), de 28 até 36 anos $(29,1 \%)$ e pessoas com 37 anos ou mais (15\%). Na Figura 2, pode-se observar o desempenho dos sujeitos em relação à faixa etária e à pontuação obtida na medida concentração do AS.

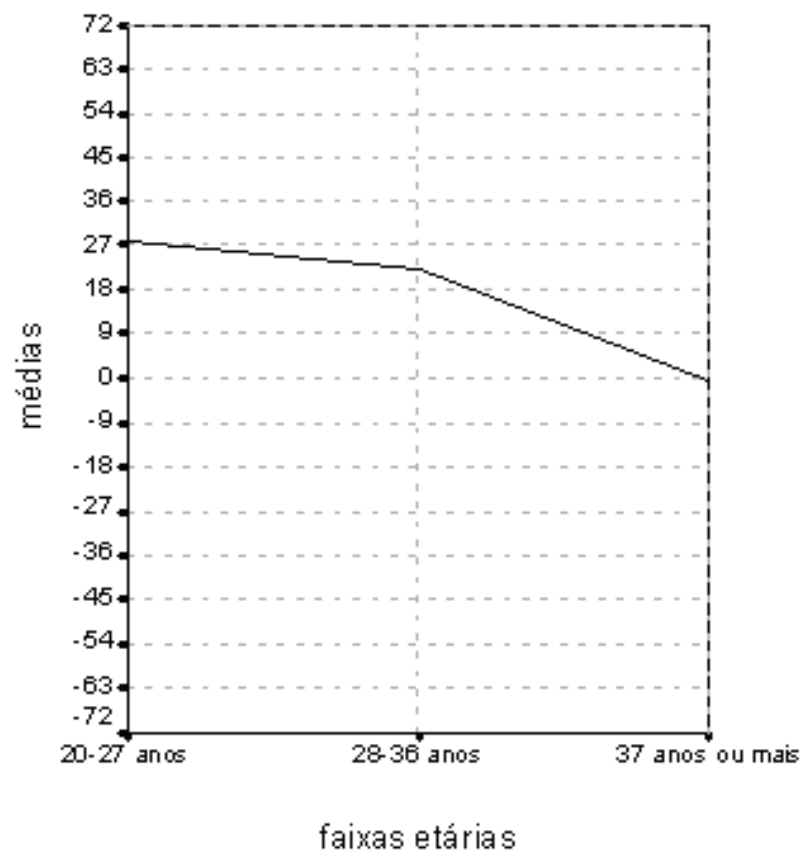

Figura 2. Médias das pontuações em concentração por faixas etárias dos participantes. 
Em relação à medida de velocidade com qualidade, foi utilizado o mesmo procedimento que na medida concentração. A distribuição das médias pelas idades está representada na Figura 3, por meio de um gráfico linear.

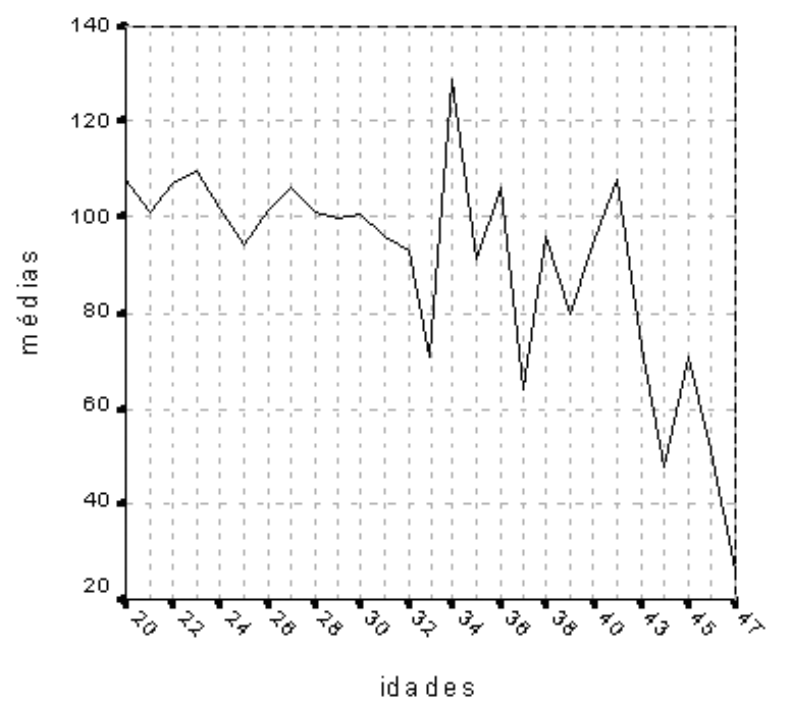

Figura 3. Média das pontuações na medida velocidade com qualidade no AS por idade dos participantes.

No caso da velocidade com qualidade, foram formadas as mesmas faixas etárias que na medida de concentração. O desempenho dos sujeitos pode ser visualizado na Figura 4.

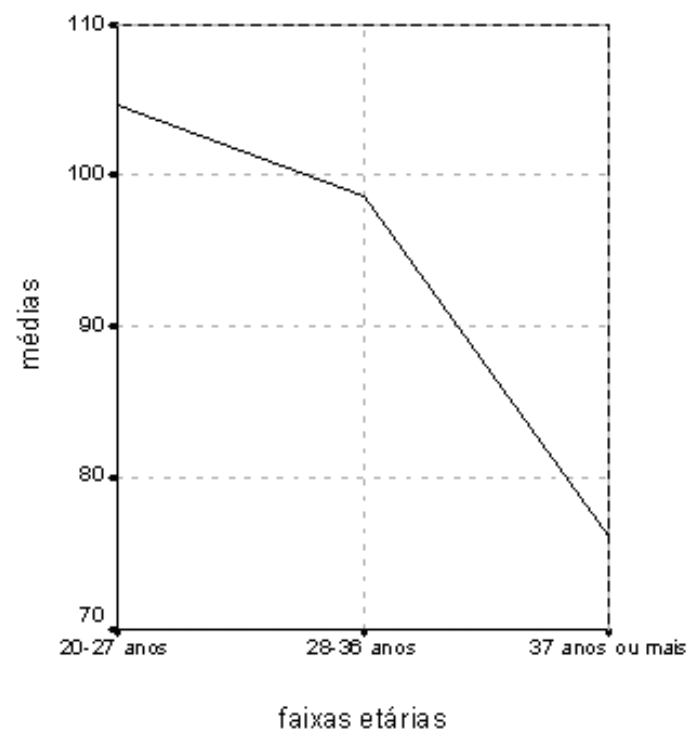

Figura 4. Médias das pontuações em velocidade com qualidade por faixas etárias dos participantes.

Verifica-se, nas Figuras 3 e 4, que, conforme aumentou a faixa etária, diminuiu a concentração e a velocidade da qualidade. Isso vai ao encontro do que é apresentado do manual do teste em relação às faixas etárias trabalhadas. 
Em seguida, são apresentadas as análises de variância das medidas estudadas no AS com as faixas etárias formadas neste estudo. Os resultados podem ser observados na Tabela 1.

Tabela 1. Valores de $F$ e $p$ obtidos pela análise de variância entre as medidas do AS e a faixa etária dos participantes.

\begin{tabular}{lcc}
\hline & $F$ & $p$ \\
\hline Concentração & 10,01 & 0.000 \\
Velocidade com qualidade & 9,54 & 0.000 \\
\hline
\end{tabular}

Como sumariado na Tabela 1, a concentração e a velocidade com qualidade apresentaram diferenças significativas entre os grupos de idade formados. Vale ressaltar que, embora tenham sido observadas tais diferenças, os valores de $F$ foram bastante menores que os apresentados no manual do teste. Quando realizada a prova de Tukey para verificar o resultado de quais faixas etárias poderiam explicar essas diferenças, observou-se que o grupo de pessoas com 37 anos ou mais foi diferenciado tanto na medida de concentração quanto na de velocidade com qualidade.

\section{Discussão}

O estudo teve como objetivo fornecer evidência de validade de construto para o Teste de Atenção Sustentada (Sisto et al., 2006). Num primeiro momento, foram apresentadas as estatísticas descritivas para as medidas fornecidas pelo AS, verificando-se que, tanto na concentração quanto na velocidade com qualidade, as pontuações, de forma geral, tenderam a ser altas.

Quando correlacionadas as medidas do teste com a idade, verificaram-se correlações que se aproximaram de -0,40, o que indica a existência de uma comunalidade de aproximadamente $16 \%$ entre a concentração e a velocidade com qualidade e a idade; ainda, as correlações foram praticamente iguais para ambas as medidas. Quando comparado esse resultado com o manual do teste (Sisto et al., 2006), observa-se que nele são apresentadas correlações consideravelmente maiores para ambas as medidas ( $r=-0,51$ e $r=-0,57)$ para a concentração e a velocidade com qualidade, respectivamente.

Posteriormente, foram criadas categorias de idades, formando-se três grupos de faixas etárias, em consonância com as apresentadas pelo manual do teste. Com base nessas categorias, foi verificado, por meio de gráficos lineares, que, quanto maior a faixa etária, menor a pontuação na concentração e na velocidade com qualidade. Esses dados confirmam os resultados encontrados por Ribaupierre e Ludwig (2003), no sentido de que pessoas com idade mais avançada apresentam maior dificuldade em tarefas que apresentam mais dificuldade, assim como também os resultados encontrados por Anderson et al. (2000), que, ao estudarem a performance numa tarefa de atenção em jovens e adultos velhos, verificaram um melhor desempenho nos jovens. Por sua vez, os resultados contrastam com a pesquisa de Berardi, Parasuraman e Haxby (2001), que não encontraram diferenças significativas na atenção sustentada em função das faixas etárias. 
Na análise de variância realizada, as diferenças observadas pelos gráficos lineares apresentaram índices de significância menores que 0,05 , no caso das medidas de concentração e de velocidade com qualidade, o que indicou que esses resultados não poderiam ser atribuídos ao acaso. A prova de Tukey ainda diferenciou as faixas etárias em grupos, sendo que, em todos os casos, os indivíduos com 37 anos ou mais foram separados dos outros grupos de pessoas.

Se levado em consideração o que foi apontado por Potvin et al. (1988), de que a experiência na estrada estaria associada a um menor envolvimento em acidentes, pode-se inferir que uma baixa performance em concentração e velocidade com qualidade associada a uma idade avançada poderia ser um indicativo para que maiores cuidados sejam tomados ao se conceder a carteira de habilitação nesses casos. Em contrapartida, como apontam Preusser et al. (1985), o maior número de acidentes acontece com pessoas jovens, o que possibilita a inferência de que os diferentes mecanismos envolvidos no ato de dirigir sejam investigados, como, por exemplo, personalidade, inteligência e memória, dentre outros construtos, uma vez que apenas a atenção pode não ser um indicativo conclusivo.

Resumindo, este estudo tende a contribuir para o desenvolvimento da área, fornecendo mais uma evidência de validade de construto para o Teste de Atenção Sustentada. Sugerese, no entanto, que outras investigações sejam feitas, utilizando-se amostras e instrumentos distintos. 


\section{Fabián Javier Marín Rueda *}

Psicólogo, perito em avaliação psicológica, Mestre em Psicologia e doutorando em Avaliação Psicológica pela Universidade São Francisco. É bolsista da CAPES.

\section{Ana Paula Porto Noronha}

Doutora em Psicologia, Ciência e Profissão pela Pontifícia Universidade Católica de Campinas e docente do Programa de Pós-Graduação Stricto Sensu em Psicologia da Universidade São Francisco. É bolsista de produtividade em pesquisa do CNPq.

\section{Fermino Fernandes Sisto}

Doutor pela Universidad Complutense de Madrid, Livre-docente pela Unicamp e docente do Curso de Psicologia e do Programa de Pós-Graduação Stricto Sensu em Psicologia da Universidade São Francisco. É bolsista de produtividade em pesquisa do CNPq.

\section{Daniel Bartholomeu}

Psicólogo e Mestre em Psicologia do Programa de Pós-Graduação Stricto Sensu em Psicologia da Universidade São Francisco.

\section{* Endereço para envio de correspondência:}

Rua José Marciano Filho, 09 - Bairro Vila Cristo Redentor

Itatiba - São Paulo - CEP: 13251-420

E-mail: marinfabian@yahoo.com.br

Recebido 29/06/2007 Reformulado 16/06/2008 Aprovado 15/07/2008

Anderson, N. D., Lidaka, T., Cabeza, R., \& Craik, F. I. M. (2000). The effect of divided attention on encoding and retrievalrelated brain activity: A PET study of younger and older adults. Journal of Cognitive Neuroscience, 12(5), 775-792.

Berardi, A., Parasuraman, R., \& Haxby, J. V. (2001). Overall vigilance and sustained attention decrements in healthy aging. Experimental Aging Research, 27, 19-39.

Brickenkamp, R. (2004). d2: Test de atención (2a ed.). Madrid: TEA Ediciones.

Cambraia, S. V. (2004). Teste de Atenção Concentrada. São Paulo: Vetor Editora Psicopedagógica.

Campagne, A., Pebayle, T., \& Muzet, A. (2004). Correlation between driving errors and vigilance level: Influence of the driver's age. Physiological Behaviors, 80(4), 515-524.

Chan, R. C. K. (2001). A further study on the Sustained Attention Response to Task (SART): The effect of age, gender and education. Brain Injury, 15(9), 819-829.

Crundall, D. E., \& Underwood, G. (1998). Effects of experience and processing demands on visual information acquisition in drivers. Ergonomics, 41(4), 448-458.

Gaddes, W. H., \& Edgell, D. (1994). Learning disabilities and brain function. New York: Springer-Verlag.

Kington, R., Reuben, D., Rogowski, J., \& Lillard, L. (1994). Sociodemographic and health factors in driving patterns after 50 years of age. American Journal of Public Health, 84(8), 1327-1329.

Lezak, M. D. (1995). Neuropsychological assessment (3rd ed.). New York: Oxford University Press.

Lucidi, F., Devoto, A., Bertini, M., Braibanti, P., \& Violani, C. (2002). The effects of sleep debt on vigilance in young drivers:
An education/research project in high schools. Journal of Adolescent, 25(4), 405-414.

Luria, A. R. (1979). Curso de psicologia geral. Rio de Janeiro: Civilização Brasileira.

Mira, A. G. (2004). Psicodiagnóstico miocinético. São Paulo: Vetor Editora Psicopedagógica.

Potvin, L., Champagne, F., \& Laberge-Nadeau, C. (1988). Mandatory driver training and road safety: The Quebec experience. American Journal of Public Health, 78(9), 12061209.

Preusser, D. F., Williams, A. F., \& Lund, A. K. (1985). Driver licensing age and lifestyles of 16 year olds. American Journal of Public Health, 75(4), 358-360.

Ribaupierre, A., \& Ludwig, C. (2003). Age differences and divided attention: Is there a general deficit? Experimental Aging Research, 29, 79-105.

Silva, M. F. G. (1999). Estudo comparativo sobre o desempenho em provas de memória e atenção entre mulheres prémenstruadas, deprimidas e não deprimidas. Dissertação de Mestrado, Instituto de Psicologia, Universidade de São Paulo, São Paulo.

Sisto, F. F. (2006). Teste Conciso de Raciocínio (TCR). São Paulo: Vetor Editora Psicopedagógica.

Sisto, F. F., Noronha, A. P. P., Lamounier, R., Rueda, F. J. M., \& Bartholomeu, D. (2006). Teste de Atenção Sustentada. São Paulo: Vetor Editora Psicopedagógica.

Sternberg, R. J. (2000). Psicologia cognitiva. Porto Alegre: Artmed.

Zillmer, E. A., \& Spiers, M. V. (1998). Principles of clinical neuropsychology. Pacific Groove: Brooks/Cole. 